\title{
Prediction of odd-mode instabilities under output mismatch effects
}

\author{
ALMUDENA SUÁREZ, FRANCO RAMÍREZ AND SERGIO SANCHO
}

\begin{abstract}
A methodology is presented to predict odd-mode instability in power amplifiers under output mismatch effects, as in the case of amplifiers connected to an antenna. This kind of instability is often observed in multi-device configurations, due to their symmetry properties. Unlike the single-ended situation, there is a cancellation of odd multiples of the oscillation frequency at the circuit output, so there is no impact of the load-impedance values at the sideband frequencies. The odd-mode instability only depends on the impedance terminations at the fundamental frequency and its harmonic terms, and can only be detected within the circuit unstable loop, instead of the antenna-connection terminals. The possible unstable modes are related with the eigenvectors of an outer tier conversion matrix accounting for the symmetry properties of the circuit topology. Under sufficient low-pass filtering of the amplifier output network, the analysis parameters can be limited to the magnitude and phase of the reflection coefficient at the fundamental frequency. This analysis involves a computationally efficient graphical technique to detect potential instabilities and a bifurcation-detection method to determine the instability boundaries in the Smith chart. The two main types of instability from periodic regime are considered, respectively associated with incommensurable and subharmonic oscillations. Results have been validated through pole-zero identification and experimental measurements.
\end{abstract}

Keywords: Linear and non-linear CAD techniques, Circuit design and applications

Received 21 October 2016; Revised 18 May 2017; Accepted 10 July 2017; first published online 23 August 2017

\section{INTRDDUCTION}

The instability of power amplifiers (PAs) under termination conditions other than $50 \Omega$, usually due to antenna mismatch $[1,2]$, can lead to serious malfunctioning, involving the observation of incommensurable oscillations and frequency divisions by two [2]. To guarantee a reliable operation in a variety of conditions, some applications impose stable operation even under highly reflective loads [3]. This stability analysis must be carried out under unknown values of the load impedance, which will be different from $50 \Omega$. Due to its frequency dependence, this impedance will be different at the fundamental frequency $f_{\text {in }}$ and its various harmonic components, $m f_{i n}$, and sideband frequencies, $m f_{i n}+f$, where $m$ is an integer and $f$ is a perturbation frequency, to be swept in the stability analysis $[4,5]$. Even- and odd-mode instabilities can be distinguished, which, as described next, require different analysis procedures.

In the even-mode instability, the mismatched-termination impedances at the harmonic frequencies $m f_{i n}$ and sideband frequencies $m f_{\text {in }}+f$ must be taken into account. Due to the low-pass filtering action of the output network, it will be possible to limit the mismatch effects to $f_{\text {in }}$ and the lowest sideband frequencies $f,-f_{\text {in }}+f$ and $f_{\text {in }}+f$, with all the rest of

Communications Engineering Department, University of Cantabria, Av. Los Castros s/n, 39005, Santander, Spain

Corresponding author:

F. Ramírez

Email: ramirezf@unican.es components arbitrarily terminated in $50 \Omega$. Taking this into account, the work [4] relies on the definition of a $3 \times 3$ scattering matrix at the reference plane, where the PA output is connected to the antenna. This is done considering three virtual ports, corresponding to the three mismatched sidebands, with the rest of harmonics and sideband frequencies constituting an inner tier. Under fulfillment of an equivalent of the Rollet's proviso [6] established in [4], the potential instability can be detected at PA output reference plane, using three large-signal equivalents of the stability factor $(\mu)$ [7], defined in [4]. These large-signal $\mu$ factors depend on the reflection coefficient $\Gamma_{o}$ at $f_{i n}$, the perturbation frequency $f$, and the input power $P_{\text {in }}$.

In the odd-mode stability analysis, the components at $m f_{\text {in }}+f$ are inherently cancelled at the PA output reference plane. This is due to the $180^{\circ}$ phase shift between different amplifier branches, which gives rise to a virtual short circuit at $m f_{i n}+f$ at the circuit output. There is a cancellation of the possible unstable poles with right-hand side (RHS) zeroes [8-10], so the instability cannot be detected from the circuit output plane. On the other hand, the impedance terminations at $m f_{\text {in }}+f$ will not have any impact on the potential instability, which will only depend on the terminations at $m f_{i n}$. The stability analysis must be necessarily carried out at the internal circuit nodes or loops. This situation is similar to the one described by Freitag [11] in the small-signal stability analysis of multi-device PAs. However, the analysis in [11] assumes a small-signal operation of the amplifier, though, in general, the PA will be in a non-linear regime $[10,12]$ with respect to the input source at $f_{\text {in }}$. Unlike the linearization 
with respect to the dc solution considered in [11], in the presence of the perturbation at $f$, the circuit must be linearized about the periodic steady-state periodic solution, which will depend on the input power $P_{i n}$. This linearization should be carried out with the conversion-matrix approach [13-15].

In the odd-mode case, despite the cancellation of the sideband frequencies $m f_{\text {in }}+f$ at the PA output reference plane, the antenna mismatch may lead to instability, due to the impact of the impedance terminations at the harmonic frequencies $m f_{\text {in }}$ on the periodic steady-state solution about which the circuit is linearized with the conversion-matrix approach. Under the assumption of a sufficient low-pass filtering action of the output network, the impact on the stability properties of the $\Gamma_{o}$ termination impedances at frequencies $m f_{i n}$, where $|m|>1$, can be considered negligible, even if all these frequencies, as well as the sideband frequencies $m f_{i n}+f$ are duly taken into account in the analysis. For simplicity, they are assumed to be terminated in $50 \Omega$, so the mismatch effects are limited to the termination at $f_{i n}$, given by $\Gamma_{o}$. In the absence of filtering effects, the analyses presented in this work would still be applicable, but would need the consideration of all passive termination impedances $\Gamma_{o, m}$ at the harmonic components $|m|>1$. Nevertheless, to protect the PA against mismatch-induced instability, it will be convenient to use a low-pass output network to minimize the impact of the termination impedances at $|m|>1$.

The practical analysis method presented in this work involves three different stages. In the first stage, the possible oscillation modes are identified through the eigenvalue/eigenvector analysis of the outer tier conversion matrix that describes the active subcircuit. This is defined at the nodes where this active subcircuit is connected to the powercombining network. The eigenvalue/eigenvector analysis is formally identical to the one proposed by Freitag [11]. In the second stage, a graphical method is used to detect potential instabilities associated to the various operation modes, excited with the aid of auxiliary generators (AGs) $[15,16]$. In the third stage, the fundamental-frequency terminations $\Gamma_{o}$ that give rise to instability are determined with a bifurcation [15-25] detection technique. It is taken into account that at the oscillation boundary, the steady-state oscillation condition is fulfilled for amplitude tending to zero $[15,16]$. A boundary analysis should be carried out for each of the oscillation modes detected in the first stage. The boundary is calculated in terms of the termination $\Gamma_{o}$ and traced in the Smith chart. Stable and unstable regions are distinguished through pole-zero identification [8-10], applied to representative points in the distinct regions separated by the boundary.

The odd-mode instability often involves a subharmonic oscillation due to the influence of the input signal at $f_{\text {in }}$ on the critical circuit frequencies, which are shifted to the divided-by-two frequency $f_{\text {in }} / 2$ [18]. Therefore, two cases will be distinguished: an incommensurable oscillation at a frequency $f$ and a subharmonic oscillation at $f_{\text {in }} / 2$. The PA operates in a large-signal regime, so the input power will have a significant impact on the potential-instability properties, which will be investigated in detail with a graphical technique. The method will be illustrated through its application to two power-combining amplifiers at $f_{\text {in }}=1.5 \mathrm{GHz}$, with two and four active devices, respectively, which have been manufactured and measured.

The paper is organized as follows. Section II presents the methodology for the detection of odd-mode instabilities, illustrated with a basic PA cell of two transistor devices. The impact of input power is analyzed in detail. Section III describes the stabilization method. Section IV presents the generalization of the potential-instability analysis to multidevice PAs.

\section{DETECTIDN DF DDD-MDDE INSTABILITIES}

Let a circuit exhibiting symmetries, such as the one in Fig. 1, be considered. For the analysis of mismatch effects, and assuming sufficient low-pass filtering effects of the output network, the circuit will be terminated in $\Gamma_{o}$ at $f_{i n}$ and in $50 \Omega$ at $m f_{\text {in }}$, where $|m|>1$. For instance, in Fig. 1 , a $180^{\circ}$ shorted stub at $2 f_{\text {in }}$ has been introduced in parallel with the final $50 \Omega$ load (the load that will undergo changes under the mismatch effects), which should help reduce the impact of mismatch at frequencies with $|m|>1$. For a given input power $P_{i n}$ and termination $\Gamma_{o}$, the circuit exhibits the steady-state solution $\bar{X}_{o}$, which is the vector of harmonics of the various state variables, obtained with harmonic balance (HB).

For a simple analysis of the impact of the circuit topology on the possible oscillation modes, an exemplary outer tier conversion matrix will be calculated. An admittance matrix is obtained by sequentially connecting an AG of voltage type $[15,16]$ at the frequency $f$, in parallel at the two analysis nodes [ 1 and 2 in Fig. 1(a)]. The AG should operate in small signal and include a bandpass filter at $f_{A G}=f$. To obtain the first (second) matrix row, the AG is connected to node 1 (2), calculating the ratio between the currents, $I_{1}$ and $I_{2}$, entering the active network from node 1 and node 2 , at the particular sideband $k f_{i n}+f$ frequency, and the $A G$ voltage. In the case of the circuit in Fig. 1, the resulting matrix has the form:

$$
\left[\begin{array}{cc}
Y_{a, 11}\left(f, k f_{i n}+f\right) & Y_{a, 12}\left(f, k f_{i n}+f\right) \\
Y_{a, 12}\left(f, k f_{i n}+f\right) & Y_{a, 11}\left(f, k f_{i n}+f\right)
\end{array}\right]
$$

where the subindex " $a$ " stands for "active network". Due to the circuit symmetry, the form of the outer tier conversion matrix will be the same, no matter the choice of the outer tier sidebands. This matrix topology exhibits the two eigenvectors $\left[\begin{array}{ll}1 & 1\end{array}\right]^{T}$ and $\left[\begin{array}{ll}1 & -1\end{array}\right]^{T}$, as easily derived from (1). On the other hand, the form of the admittance matrix describing the passive network (Fig. 1) is identical to (1) at all frequencies $m f_{\text {in }}+f$, and therefore exhibits the same two eigenvectors $\left[\begin{array}{ll}1 & 1\end{array}\right]^{T}$ and $\left[\begin{array}{ll}1 & -1\end{array}\right]^{T}$. The eigenvector $\left[\begin{array}{ll}1 & 1\end{array}\right]^{T}$ corresponds to an even mode, with the sidebands $m f_{i n}+f$ in phase in the two amplifier branches. The eigenvector $\left[\begin{array}{ll}1-1\end{array}\right]^{T}$ corresponds to an odd mode, having sidebands with $180^{\circ}$ phase shift in the two branches.

The two modes can be excited by simultaneously connecting the two voltage AGs in parallel at the two analysis nodes, at the frequency $f$, with identical amplitudes $V_{A G}$ [see Fig. 1(a)]. The mode $\left[\begin{array}{ll}1 & 1\end{array}\right]^{T}$ is analyzed by setting equal phase values at the two AGs, $\phi_{A G 1}=\phi_{A G 2}=0^{\circ}$. Under this simultaneous excitation, the total admittance at $f$, at each of the two analysis nodes is given by the ratio between each AG current and voltage: $Y_{A G_{1, e}}=I_{A G_{1}}(f) / V_{A G}, Y_{A G_{2}, e}=$ $I_{A G 2}(f) / V_{A G}$. Because the excitation agrees with one of the 


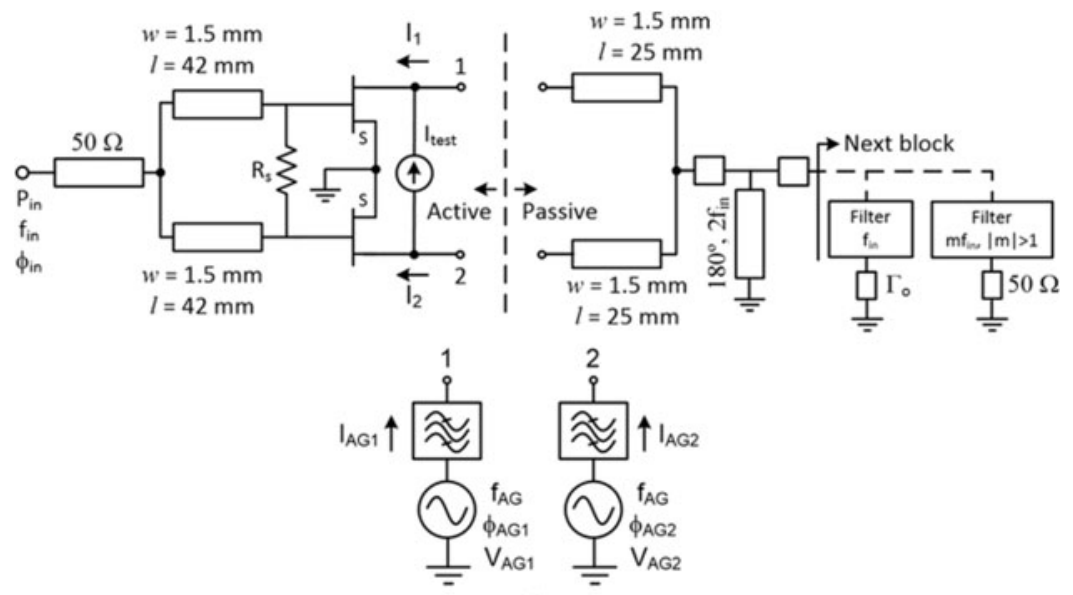

(a)

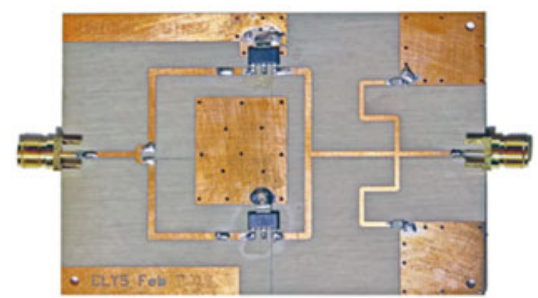

(b)

Fig. 1. Test-bench power amplifier based on a CLY5 transistor $\left(\mathrm{RO}_{4003} \mathrm{C}: \varepsilon_{\mathrm{r}}=3.38, h=0.5 \mathrm{~mm}\right)$. (a) Circuit schematic. The AGs, at the respective phases $\phi_{A G 1}$ and $\phi_{A G_{2}}$, are used for the analysis of the modes. The stabilization resistor $R_{s}$, connected between the two amplifier branches, is also shown. The small-signal current source is introduced to evaluate the limit-oscillation conditions at an incommensurable frequency $f$ or a subharmonic frequency $f_{i n} / 2$. (b) Photograph.

matrix eigenvectors, the two admittances are identical, as demonstrated in [11]. This admittance will be renamed $Y_{T e}=Y_{A G 1, e}=Y_{A G 2, e}$. In turn, the mode $[1-1]^{T}$ is analyzed by using two small-signal AGs with equal amplitude and $180^{\circ}$ phase shift, $\phi_{A G 1}=0^{\circ}, \phi_{A G 2}=180^{\circ}$. Under this simultaneous excitation, the total admittances $Y_{A G_{1,0}}=I_{A G_{1}}(f) /$ $V_{A G}$ and $Y_{A G 2, e}=I_{A G 2}(f) / V_{A G} e^{j \pi}$ are also equal, and will be denoted as $Y_{T o}$. This use of multiple AGs to analyze the modes will be most useful in the general case of multi-device PAs, as shown in Section IV.

In the case of odd-mode instability, the two transistor branches will exhibit $180^{\circ}$ phase shift at $m f_{\text {in }}+f$, so these sidebands will inherently cancel out at the PA output reference plane. This situation in which the stability properties depend on the termination at $m f_{\text {in }}$ but are independent on the terminations at the sideband frequencies at $m f_{\text {in }}+f$ can be interpreted as a failure of the proviso established in [4] (an extension of Rollet's proviso [6] to the sideband-impedance problem). This is because the odd-mode instability will be observed even if the sideband frequencies are terminated in open or short circuits at the PA output [4]. The antenna mismatch will only affect the stability properties through the termination impedances at $m f_{i n}$, which will give rise to a change in the steady-state solution $\bar{X}_{o}$ about which the circuit is linearized, and, therefore, to a change in $Y_{T o}$. However, under the assumption of sufficient low-pass filtering effects, the analysis can be limited to the termination at $f_{i n}$, expressed as $\Gamma_{o}$. The test bench is illustrated in Fig. 1, where harmonics $|m|>1$ are arbitrarily terminated in $50 \Omega$ due to their limited influence.

The prediction of the odd-mode instability will be based on two fundamental properties: (i) when a circuit exhibits a steady-state oscillation, all its nodes exhibit a total admittance function (current-to-voltage ratio) equal to zero, (ii) at the instability boundary, the steady-state oscillation amplitude tends to zero [18]. When linearizing the circuit with respect to the perturbation at $f$ (as done in the conversion-matrix approach), we implicitly assume amplitude tending to zero at this frequency. Thus, the limit oscillation condition (instability boundary) is defined by the condition: $Y_{T o}=0$. This odd-mode limit oscillation condition can also be evaluated through the connection of a small-signal current source between equivalent device nodes of the two subcircuits (Fig. 1), doing $Y_{T o}=I_{\text {test }} t$ $\left(V_{1}-V_{2}\right)$. Note that $Y_{T o}$ has been redefined here.

Pole-zero identification [8-10] would be applicable to detect the odd-mode instabilities under mismatch effects. However, the odd-mode excitation must be preserved as otherwise the impedance at sideband frequencies would be ill represented with a constant $\Gamma_{o}$. Such an analysis would require a sweep in the perturbation frequency $f$ (going from o to $f_{i n}$, in periodic regimes) for each steady-state solution, obtained through a double sweep in the amplitude and phase of $\Gamma_{o}$. Pole-zero identification should be applied to all the transfer functions resulting from this double sweep. The identification interval o to $f_{\text {in }}$ should be, in general, divided into smaller intervals, so this analysis will be computationally demanding. Instead, the aim here will be to obtain directly the boundary between stable and unstable values of $\Gamma_{o}$, given by the condition $Y_{T o}=0$, which should be traced in the Smith chart corresponding to $\Gamma_{o}$. Actually, the fulfillment of $Y_{T o}=$ o will depend on the input power $P_{i n}$, the termination $\Gamma_{o}$, and the oscillation characteristics, since there are two major instability mechanisms from a periodic regime at $f_{\text {in }}$ [1520]. One is the onset of an incommensurable oscillation at the frequency $f$, or Hopf bifurcation, and the other is a division by 2 of the input frequency, leading to a subharmonic regime at $f_{\text {in }} / 2$, which corresponds to a flip bifurcation. 
For illustration, the analysis will be applied to the PA in Fig. 1. Introducing a stabilization resistor, $R_{s}=170 \Omega$, in parallel between the two amplifier branches, the circuit is stable for all the $P_{i n}$ values under matched conditions (when terminated in a $50 \Omega$ load), as has been verified with pole-zero identification [8-10]. On the other hand, the amplifier does not exhibit even-mode instabilities under mismatch effects, as verified with the method in [4].

The analysis method relies on the calculation of the Hopfand flip-bifurcation loci, in terms of the magnitude and phase of the reflection coefficient $\Gamma_{o}$. This must be complemented with a local-stability analysis, such as pole-zero identification [8-10] to distinguish between stable and unstable regions. It will be sufficient to apply this local-stability analysis to representative points in the various regions separated by the loci.

\section{A) Incommensurable oscillation}

For each $P_{i n}$, the boundary of incommensurable oscillations, or Hopf-bifurcation locus [15-20], will be defined by the two conditions:

$$
\begin{aligned}
\bar{H}\left(\bar{X}_{o}, \rho_{o}, \phi_{o}\right) & =0, \\
Y_{T o}\left(\bar{X}_{o}, \rho_{o}, \phi_{o}, f\right) & =0,
\end{aligned}
$$

where $\bar{H}=$ o represents the whole set of $\mathrm{HB}$ equations, acting as an inner tier, $\rho_{o}, \phi_{o}$ are the magnitude and phase of $\Gamma_{o}$ and $f$ is incommensurable with $f_{i n}$. The steady-state solution $\bar{X}_{o}$ depends on $\Gamma_{o}$ and the limit oscillation condition, $Y_{T o}=0$, is evaluated with the conversion-matrix approach.

The analysis based on (2) should start with a global exploration of the Smith chart, in order to provide a suitable initial value to the optimization/calculation procedure. This is done with a simple graphical technique that takes advantage of the bounded nature of $\rho_{o}$ and $\phi_{o}$. The perturbation frequency $f$ is swept between o and $f_{\text {in }}$ [8-10] and, for each $f$, a double sweep is performed in $\rho_{o}, \phi_{o}$, so as to cover the entire Smith chart. For each triplet $f, \rho_{o}, \phi_{o}$, the total admittance $Y_{T}$ is calculated as the ratio between the current delivered by the small-signal source and the voltage across its terminals $Y_{T}=I_{\text {test }} /\left(V_{1}-V_{2}\right)$. A closed curve is obtained for each pair of values $f, \rho_{o}$. To fulfill $Y_{T o}=0$, there must be changes of sign in both the real and imaginary parts of $Y_{T}$ under variations of $\rho_{o}, \phi_{o}$, which is easily evaluated through simple inspection.

Figure 2(a) shows the admittance plots versus $f, \rho_{o}, \phi_{o}$ corresponding to the circuit in Fig. 1, obtained for $P_{\text {in }}=10 \mathrm{dBm}$. There are no crossings of the negative real semi-axis, so there is no oscillation boundary within the Smith chart. Because the PA is always stable in matched conditions, the value $\Gamma_{o}=0$ is stable and, as a result, the whole Smith chart should be stable too. The same situation is obtained for other $P_{\text {in }}$ values, so one concludes that the PA in Fig. 1 cannot exhibit incommensurable oscillations under mismatch effects.

In case the presence of an instability boundary is detected, suitable initial values for the calculation of this boundary should be close to the origin of the admittance plane $\left(Y_{T}=o\right)$. This situation may be found in one or several regions of the Smith chart. This initial value (or values) should be introduced in system (2), which will provide an initial point of the instability boundary: $\rho_{o}^{i}, \phi_{o}^{i}, f^{i}$. Then the entire Hopf locus will be efficiently traced through continuation [18], by sweeping $\phi_{o}$ from $\phi_{o}^{i}$ and solving (2) to obtain: $\rho_{o}\left(\phi_{o}\right), f\left(\phi_{o}\right)$. There will be one Hopf locus for each $P_{i n}$.

\section{B) Frequency division by 2}

One common case of odd-mode instability is the frequency division by 2 , associated with flip bifurcations [13-20]. This phenomenon occurs when the input signal shifts the circuit natural frequency $f$ to one-half of the input frequency: $f \rightarrow f_{\text {in }} / 2$, which is often associated with a parametric instability. This evolution involves the splitting of a pair of complex-conjugate poles at $f$ (associated with two dimensions of the differential equation system [18]) into two independent pairs of complex-conjugate poles at $f_{\text {in }} / 2$ (each associated with one dimension). At the division threshold, the subharmonic-oscillation amplitude will tend to zero, so the flip bifurcations can be detected by setting the frequency of the small-signal current source to $f_{\text {in }} / 2$. Because this perturbation frequency $\left(f_{i n} / 2\right)$ and the input frequency are commensurable, the phase shift between the input source and the current source is a relevant analysis variable [19]. For the bifurcation detection, one can set the phase of the current source to zero and consider the input-source phase $\phi_{\text {in }}$ as an analysis variable. The mathematical conditions for the flip bifurcation are:

$$
\begin{aligned}
\bar{H}\left(\bar{X}_{o}, \rho_{o}, \phi_{o}, \phi_{i n}\right) & =0, \\
Y_{T o}\left(\bar{X}_{o}, \rho_{o}, \phi_{o}, \phi_{i n}\right) & =0,
\end{aligned}
$$

where $\bar{H}=\mathrm{o}$ is the whole set of $\mathrm{HB}$ equations, acting as an inner tier, and $\rho_{o}, \phi_{o}$ are the magnitude and phase of $\Gamma_{o}$. The steady-state solution $\bar{X}_{o}$ depends on $\Gamma_{o}$. Unless a modified conversion-matrix analysis $[21,22]$ is applied, the above analysis must be carried out with $\mathrm{HB}$ at the fundamental frequency

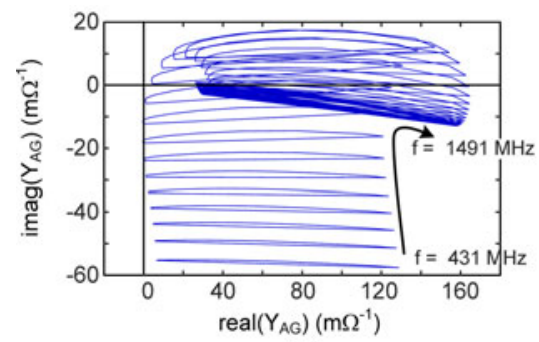

(a)

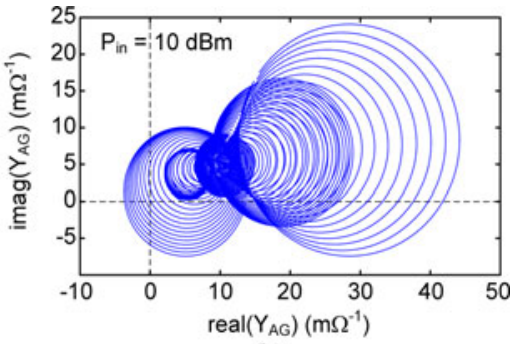

(b)

Fig. 2. Graphical method to obtain initial values, using the total admittance function. (a) Results in the case of an incommensurable frequency $f$, sweeping in $f$, $\rho_{o}$, and $\phi_{o}$ at the constant input power $P_{i n}=10 \mathrm{dBm}$. No instability boundary can exist within the unit Smith chart. (b) Results in the case of a subharmonic frequency $f_{\text {in }} / 2$ and $P_{\text {in }}=10 \mathrm{dBm}$, sweeping $\phi_{i n}, \rho_{o}$, and $\phi_{o}$. There should be an instability boundary inside the unit Smith chart. 


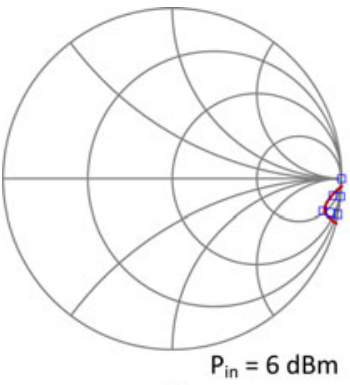

(a)

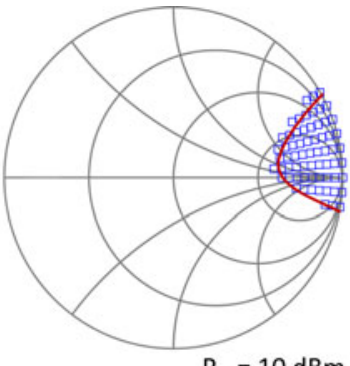

(b)

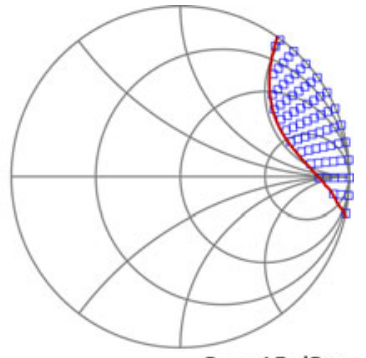

$P_{\text {in }}=15 \mathrm{dBm}$

(c)

Fig. 3. Instability boundaries, showing also the points fulfilling $\operatorname{Re}\left(Y_{T o}\right)<\mathrm{o}$ and $\left|\operatorname{Im}\left(Y_{T o}\right)\right| \leq 10^{-3} \Omega^{-1}$. (a) For $P_{\text {in }}=6 \mathrm{dBm}$. (b) For $P_{\text {in }}=10 \mathrm{dBm}$. (d) For $P_{\text {in }}=15 \mathrm{dBm}$.

$f_{\text {in }} / 2$, due to the frequency commensurability. The initial value(s) is obtained through three nested sweeps, in the inputsource phase $\phi_{i n}$, varied between o and $360^{\circ}$, and in $\rho_{o}, \phi_{o}$, to cover the full Smith chart. Advantage is taken from the fact that the three sweeps are bounded.

In the case of the PA in Fig. 1, the admittance plots $Y_{T}\left(\bar{X}_{o}, \rho_{o}, \phi_{o}, \phi_{i n}\right)$ provide several crossings of the negative real semi-axis [Fig. 2(b)], indicating the fulfillment of the flipbifurcation condition (3) for a continuous set of triplets $\rho_{o}, \phi_{o}$, $\phi_{i n}$. Once an initial point $\rho_{o}^{i}, \phi_{o}^{i}, \phi_{i n}^{i}$ has been obtained, the entire instability boundary is easily traced through continuation, by sweeping the phase $\phi_{o}$ from $\phi_{o}^{i}$, and solving (3) to obtain: $\rho_{o}\left(\phi_{o}\right), \phi_{i n}\left(\phi_{o}\right)$. This provides the flip-bifurcation loci shown in Fig. 3, which constitute the boundary of load-impedance values for which the circuit exhibits a subharmonic oscillation (flip-bifurcation locus). Each locus in Fig. 3 corresponds to a different $P_{i n}$. The stable and unstable regions of this boundary are easily distinguished since one should know beforehand the stability properties of the matched amplifier, that is, when it is terminated in $50 \Omega$. Because the amplifier is stable in matched conditions, the stable region corresponds to the outside of the flip loci.

Processing the data in Fig. 2(b), it has been possible to obtain the $\Gamma_{o}$ values giving negative conductance $\left(\operatorname{Re}\left(Y_{T}\right)<0\right)$, with a magnitude of the imaginary part $\left|\operatorname{Im}\left(Y_{T}\right)\right|$ below $10^{-3} \Omega^{-1}$ at different $P_{\text {in }}$ values, represented with squares in Figs 3(a)-3(c). The unstable region contains a subset of the points with negative real part of $Y_{T}$ and low magnitude of the imaginary part. Note that the negative real part and positive-slope resonance of $Y_{T}$ do not constitute a general instability condition. However, the limit steady-state oscillation condition in (2) and (3) is rigorous and should be fulfilled at any circuit node at the stability boundary.

As shown in Fig. 4, for low $P_{i n}$, the flip locus does not enter the Smith chart, so there is unconditional stability. From $P_{i n} \cong$ $5 \mathrm{dBm}$, the locus crosses the Smith chart, so the amplifier is potentially unstable under mismatch effects. Due to the natural reduction of the negative resistance from certain signal amplitude, one should expect the loci to escape from the Smith chart from a certain $P_{\text {in }}$ value.

Because the odd-mode instability only depends on the load value $\Gamma_{o}$ at $f_{i n}$, all the possible implementation of this load should give rise to the same kind of behavior, either stable or unstable. This has been validated for two different $\Gamma_{o}$ values, one at each side of the flip-bifurcation locus obtained for $P_{i n}=15 \mathrm{dBm}$ (in a solid red line in Fig. 3), indicated as $\Gamma_{t_{1}}$ and $\Gamma_{t_{2}}$ in Fig. 4. They are relatively close to the stability boundary to evaluate the degree of accuracy. Figure 5(a) presents the results of an independent stability analysis based on pole-zero identification when $\Gamma_{t_{1}}$ and $\Gamma_{t_{2}}$ are implemented with an RL series network. Figure 5 (b) presents the results of the parallel-RL implementation. Poles of the $\Gamma_{t_{1}}$ $\left(\Gamma_{t_{2}}\right)$ load are represented with " + " (“ $x$ ”). With the two different implementations, the load $\Gamma_{t_{1}}$ is stable and the load $\Gamma_{t_{2}}$ is unstable, in agreement with results from (3).

As stated, to protect the PA against mismatch-induced instabilities, it is convenient to use a low-pass output network, as in the case of the amplifiers considered in this work. In the absence of output filtering effects, the same procedure should be applied for each particular set of reflectioncoefficient values at the harmonic frequencies $|m|>1$, which would provide a family of Hopf and flip loci. Actually, the flip locus obtained above corresponds to the case $\Gamma_{m}=0$ for $|m|>1$. For unconditional stability, the PA should be stable in matched conditions and none of the loci should enter the Smith chart. If the goal is just to check whether the PA is unconditionally stable or not, it will be sufficient to inspect the admittance diagrams obtained through consecutive sweeps. To be more precise, for each $f$ (in the case of a Hopf-bifurcation detection) or $\phi_{\text {in }}$ (in the case of flipbifurcation detection) a series of nested sweeps are carried out in the magnitude and phase of the reflection coefficients $\Gamma_{m}$, where $|m| \geq 1$. To fulfill $Y_{T o}=0$, there must be changes of sign in both the real and imaginary parts of $Y_{T}$ under the variations of $\Gamma_{m}$, which is easily evaluated through simple inspection.

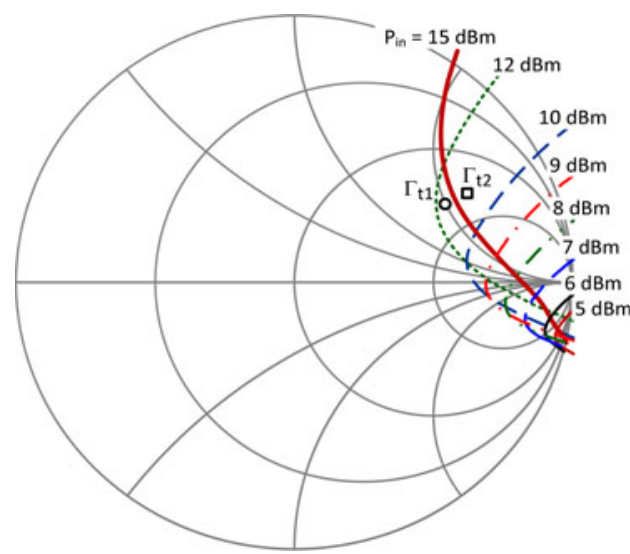

Fig. 4. Evolution of the flip locus obtained with (3) versus $P_{i n}$. The loci only cross the Smith chart in a certain $P_{i n}$ interval. The two reflection-coefficient points $\left(\Gamma_{t_{1}}\right.$ and $\left.\Gamma_{t_{2}}\right)$ at both sides of the boundary corresponding to $P_{i n}=$ $15 \mathrm{dBm}$ (used for the validation of the method) are indicated. 


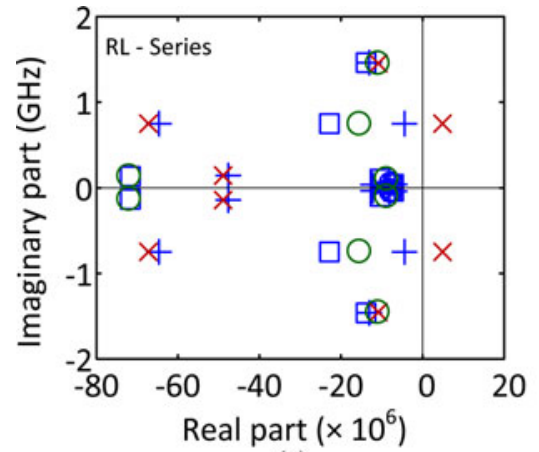

(a)

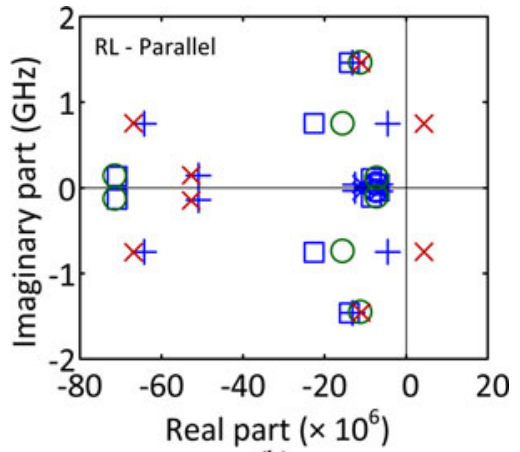

(b)

Fig. 5. Validation of the flip locus corresponding to $P_{i n}=15 \mathrm{dBm}$ with two different implementations of $\Gamma_{t_{1}}$ and $\Gamma_{t_{2}}$ in Fig. 3. (a) RL-series implementation. Poles of the $\Gamma_{t_{1}}\left(\Gamma_{t_{2}}\right)$ load are represented with “+” “ “ $\times$ ”). (b) RL-parallel implementation. Poles of the $\Gamma_{t_{1}}\left(\Gamma_{t_{2}}\right)$ load are represented with “ + " (“ $\times$ ").

\section{AMPLIFIER STABILIZATION}

The amplifier in Fig. 1 will be stabilized under mismatch effects with the aid of the resistor $R_{s}$, connected between the two amplifier branches. Because the target is to stabilize the amplifier for all the $P_{\text {in }}$ values, it will be useful to determine the $P_{\text {in }}$ interval with potential instability in an efficient manner. In the particular case of Fig. 4, all the loci cross the boundary of the Smith chart, so one can expect the locus to be tangent to this chart at the limits of the unstable $P_{\text {in }}$ interval. At the boundary of the unit Smith chart, the magnitude of the reflection coefficient is $\rho_{o}=1$. The locus of $P_{\text {in }}$ and $\phi_{o}$ values fulfilling the flip-bifurcation condition under $\rho_{o}=1$ is expressed as:

$$
\begin{array}{r}
\bar{H}\left(\bar{X}_{o}, \rho_{o}=1, \phi_{o}, \phi_{\text {in }}, P_{\text {in }}\right)=0, \\
Y_{T o}\left(\bar{X}_{o}, \rho_{o}=1, \phi_{o}, \phi_{\text {in }}, P_{\text {in }}\right)=0 .
\end{array}
$$

For $P_{\text {in }}$ values such that the flip locus in (3) crosses the unit Smith chart, there will be at least two $\phi_{o}$ fulfilling (4) (Fig. 4). This is shown in Fig. 6, where the phase $\phi_{o}$ at the intersection points with the Smith chart [calculated with (4)] has been represented versus $P_{i n}$. At the boundaries of the unstable $P_{\text {in }}$ interval, there will only be one $\phi_{o}$, since the locus is tangent to the Smith chart. To stabilize the circuit under mismatch effects, the resistor $R_{s}$, connected between the two amplifier branches, will be reduced from its original value (170 $\Omega$ ), in order to increase the damping effects in the odd mode. As expected (Fig. 6), the locus (4) decreases in size with $R_{s}$ and eventually vanishes. For $R_{s}<120 \Omega$, the amplifier should be stable for all the $P_{\text {in }}$ values.

The PA has been measured for two $R_{s}$ values (150 and $100 \Omega$ ) and different positions of a triple-stub tuner, used to enable the load variation [Fig. $7(\mathrm{a})$ ]. With $R_{s}=150 \Omega$, the circuit is stable for the measured loads $A$ and $B$ and exhibits a frequency division by 2 for the loads $C$ and $D$. See the spectra corresponding to $B$ and $C$ in Figs $7(b)$ and $7(c)$. The low amplitude of the subharmonic spectral line is due to the near cancellation of this frequency component at the circuit output, due to its odd-mode nature. The region of the unstable loads is in very good correspondence with the analysis in Fig. 4. With $R_{s}=100 \Omega$, the circuit is stable for all the load values [E, F, G, $H$ are shown in Fig. 7(a)] and all the $P_{\text {in }}$ values, in agreement with Fig. 6 .

\section{GENERAL METHDDDLDGY FDR THE PREDICTION DF POTENTIAL DDD-MDDEINSTABILITIES}

The methodology in Section II can be generalized to multidevice PAs, exhibiting symmetries. According to [11], in general, there are $N$ possible modes when $N$ devices are combined. The method to detect mismatch-induced instabilities should consider all of the possible modes, detected through the eigenvalue/eigenvector analysis [11] of the outer tier conversion matrix describing the active subcircuit. Each of them should be tested for the two cases of incommensurable and subharmonic oscillations.

For instance, the PA in Fig. 8 is a two-stage extension of the one in Fig. 1, containing four transistor devices. Under matched conditions ( $50 \Omega$ termination), with the stabilization resistor $R_{s}=100 \Omega$ connected as shown in Fig. 9(a), this PA exhibits unstable behavior in a certain $P_{i n}$ interval, as observed when applying pole-zero identification [8-10]. Figure 9 shows the evolution of the real part of the dominant poles versus $P_{i n}$. For low $P_{i n}$, there is a pair of dominant complex-conjugate poles at a frequency $f$, which is incommensurable with the drive frequency $f_{i n}$. The poles are initially located on the lefthand side (LHS) of the complex plane, but, as $P_{\text {in }}$ increases, they approach the imaginary axis and cross this axis to the RHS at $P_{i n 1} \cong 2.55 \mathrm{dBm}$. At $P_{i n 2} \cong 10.9 \mathrm{dBm}$, the same pair of complex-conjugate poles crosses to the LHS and the amplifier becomes stable. Thus, the matched amplifier is unstable in the input power interval $(2.55,10.9 \mathrm{dBm})$. To illustrate the possibilities of the new methodology, instead of a two-stage

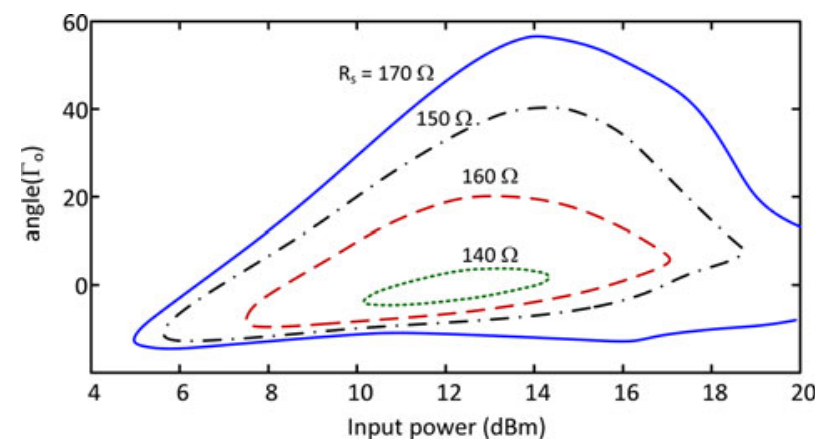

Fig. 6. Calculation of the unstable $P_{i n}$ interval using the locus in (4). The limits of this interval correspond to the edge points of the locus. The calculation has been performed for different values of the stabilization resistor $R_{s}$. 


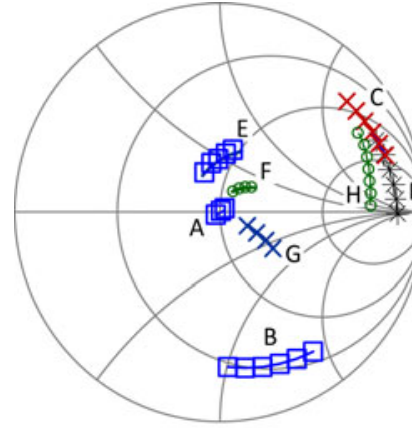

(a)

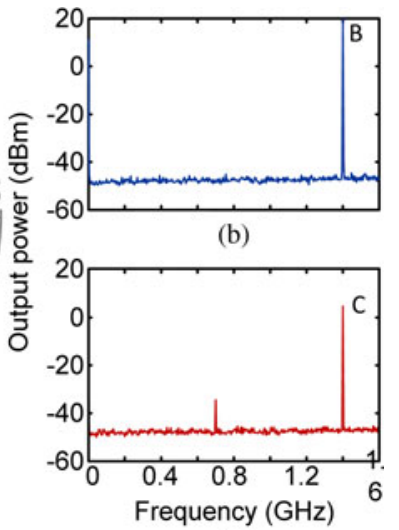

(c)
Fig. 7. Measurements for different positions of a triple-stub tuner, connected to the PA output. (a) The loads $A, B, C, D$ correspond to tests under $R_{s}=$ $150 \Omega$. The loads $E, F, G, H$ correspond to tests under $R_{s}=100 \Omega$. (b) Spectrum for $R_{s}=150 \Omega$ and load $B$ (stable). (c) Spectrum for $R_{s}=150 \Omega$ and load $C$ (unstable).

procedure, with a first stage devoted to the stabilization of the matched PA and a second one devoted to its stabilization under mismatch effects, a global stabilization process will be applied, considering all passive terminations $\Gamma_{o}$ at $f_{i n}$.

The method described in Section II to obtain an outer tier conversion-matrix approach can be generalized to PAs containing any number of active devices. A small-signal voltage AG at the perturbation frequency $f$ is sequentially connected in parallel at each of the device nodes. Then, the elements of each row of the admittance-type matrix are given by the ratio between the current entering each device at the particular sideband $k f_{i n}+f$ and the AG voltage. In the particular case of

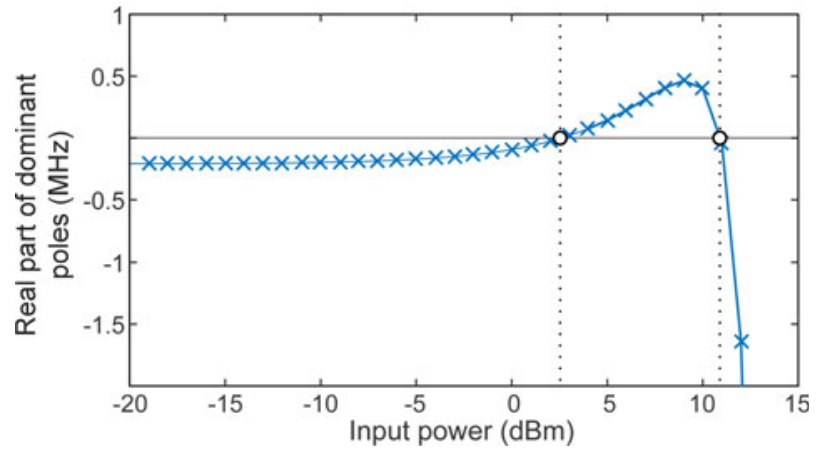

Fig. 9. Stability analysis of the PA terminated in a standard $50 \Omega$ load, with a stabilization resistor $R_{s}=100 \Omega$, connected as shown in Fig. 8. The real part of the dominant poles has been represented versus $P_{i n}$. The frequency $f$ of these poles is incommensurable with the input frequency $f_{i n}$. The PA is unstable in the interval comprised between $P_{i n 1}=2.55 \mathrm{dBm}$ and $P_{i n 2}=10.9 \mathrm{dBm}$.

four active devices, considered in Fig. 8, the matrix is expressible as:

$$
\left[\begin{array}{llll}
Y_{11}\left(f, k f_{\text {in }}+f\right) & Y_{12}\left(f, k f_{\text {in }}+f\right) & Y_{23}\left(f, k f_{\text {in }}+f\right) & Y_{23}\left(f, k f_{\text {in }}+f\right) \\
Y_{12}\left(f, k f_{\text {in }}+f\right) & Y_{11}\left(f, k f_{\text {in }}+f\right) & Y_{23}\left(f, k f_{\text {in }}+f\right) & Y_{23}\left(f, k f_{\text {in }}+f\right) \\
Y_{23}\left(f, k f_{\text {in }}+f\right) & Y_{23}\left(f, k f_{\text {in }}+f\right) & Y_{11}\left(f, k f_{\text {in }}+f\right) & Y_{12}\left(f, k f_{\text {in }}+f\right) \\
Y_{23}\left(f, k f_{\text {in }}+f\right) & Y_{23}\left(f, k f_{\text {in }}+f\right) & Y_{12}\left(f, k f_{\text {in }}+f\right) & Y_{11}\left(f, k f_{\text {in }}+f\right)
\end{array}\right]
$$

Note that the matrix structure is independent of the two sidebands chosen for the outer tier analysis. The matrix describing the passive output network at $k f_{\text {in }}+f$ will have an identical form. Thus, the possible oscillation modes can be derived from the eigenvectors of the active and passive

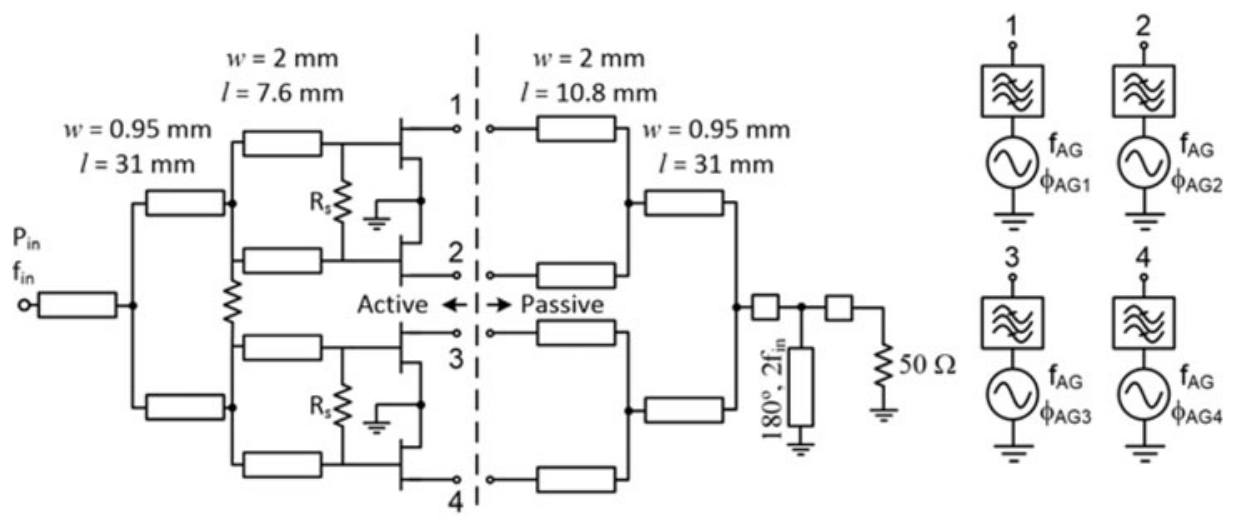

(a)

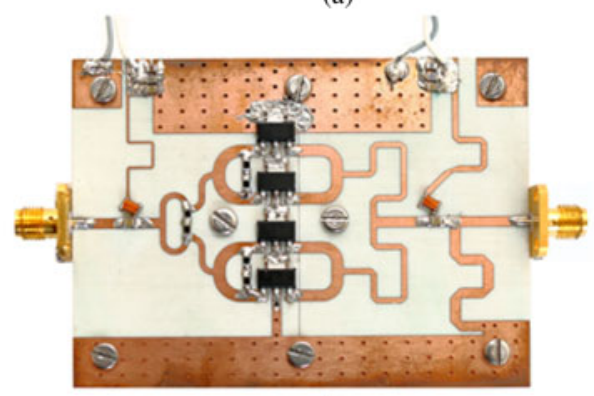

(b)

Fig. 8. PA based on four active devices, operating at $f_{i n}=1.5 \mathrm{GHz}$. (a) Schematic. The AGs used for the potential-instability analysis and operating at the frequency $f_{A G}$, are also shown. Their phases, $\phi_{A G 1}, \phi_{A G 2}, \phi_{A G 3}$, and $\phi_{A G 4}$ depend on the particular odd mode to be analyzed, as described in the main text. (b) Photograph. 
matrices. The matrix (5) (as well as the matrix describing the passive output networks) has the following eigenvectors:

$$
\begin{aligned}
& \bar{e}_{1}=\left[\begin{array}{llll}
1 & 1 & 1 & 1
\end{array}\right]^{T}, \\
& \bar{e}_{2}=\left[\begin{array}{llll}
1 & 1 & -1 & -1
\end{array}\right]^{T}, \\
& \bar{e}_{3}=\left[\begin{array}{llll}
1 & -1 & a & -a
\end{array}\right]^{T}, \\
& \bar{e}_{4}=\left[\begin{array}{llll}
-1 & 1 & -b & b
\end{array}\right]^{T} .
\end{aligned}
$$

The two last eigenvectors imply different amplitudes in the two subamplifiers. In practice, due to the symmetry of the circuit topology, one can expect identical oscillation amplitudes in all the equivalent circuit nodes. The eigenvectors in (6) indicate four possible situations, in terms of the phase shifts (o and $180^{\circ}$ ) between equivalent nodes of the four circuit branches.

Once the possible modes have been determined with the method described above, the potential-instability analysis will be based on an actual excitation of these modes through the simultaneous connection of as many voltage AGs as the number of active devices. These AGs will be connected in parallel between equivalent nodes of these devices and ground, as shown in Fig. 8(a). Note that the study can equally be carried out using current sources in parallel, since the analysis targets the instability boundary, at which the oscillation amplitude tends to zero, so the circuit behaves in linear condition with respect to these auxiliary sources.

The analysis of the possible odd-mode instabilities will consist of two different stages, depending on the desired level of insight into the potential-instability mechanism: (i) a graphical method to detect the potential-instability problem and (ii) a calculation of the stability boundaries in the Smith chart corresponding to $\Gamma_{o}$. The cases of an incommensurable oscillation and a subharmonic oscillation will be distinguished. For each, all the possible odd-mode instabilities, detected with the outer tier conversion-matrix approach, will be checked.

\section{A) Incommensurable oscillation}

For the prediction of an odd-mode incommensurable oscillation, the frequency of the AGs $\left(f_{A G}=f\right)$ must be incommensurable with the input-drive frequency $f_{i n}$.

1) MODE $\left[\begin{array}{llll}1 & -1 & 1 & -1\end{array}\right]^{T}$

To analyze the potential instability in the odd mode $\left[\begin{array}{llll}1 & -1 & 1 & -1\end{array}\right]^{T}$ four AGs, at $f_{A G}=f$, with the respective phase distributions: $\left(0,180,0,180^{\circ}\right)$, are connected in parallel at the device output terminals [Fig. 8(a)]. For each $P_{\text {in }}$ value, a triple sweep is performed, in the frequency $f$, from o to $f_{i n}$, and in the amplitude and phase of $\Gamma_{o}$. Advantage is taken from the fact that the three sweeps are bounded. For each frequency $f$ and magnitude $\rho_{o}$, a closed curve is obtained when sweeping $\phi_{o}$. Figure 10 presents the results of the triple sweeps corresponding to the mode

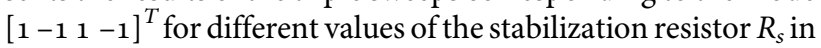
Fig. 8(a).

Figure 10 (a) shows the admittance plots with $R_{s}=100 \Omega$. For each $P_{i n}$ and each $f$, a closed curve is obtained. For $P_{\text {in }}=-10 \mathrm{dBm}$, the zero-amplitude steady-state oscillation condition $Y_{T o}\left(\bar{X}_{o}, \rho_{o}, \phi_{o}, f\right)=0$ can never be fulfilled, as derived from a simple inspection of the plots. The entire Smith chart will either correspond to stable or unstable behavior, since there is no instability boundary within this chart. From the pole-zero identification of Fig. 9, for $P_{\text {in }}=-10 \mathrm{dBm}$, the amplifier terminated in $50 \Omega$ is stable. Therefore, the entire unit Smith chart is stable and the PA is unconditionally stable under mismatch effects. The closed curves become larger for $P_{i n}=\mathrm{odBm}$, and some of them enclose the center of the complex plane [Fig. 10(a)]. As a result, a continuous set of triplets $f, \rho_{o}, \phi_{o}$ will fulfill the limit oscillation condition $Y_{T o}=0$, so there should be a stability boundary inside the $\Gamma_{o}$ Smith chart for this $P_{\text {in }}$ value. The

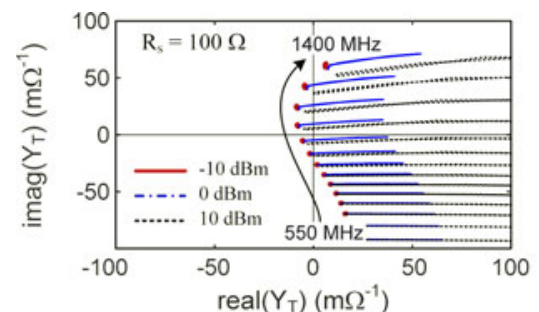

(a)

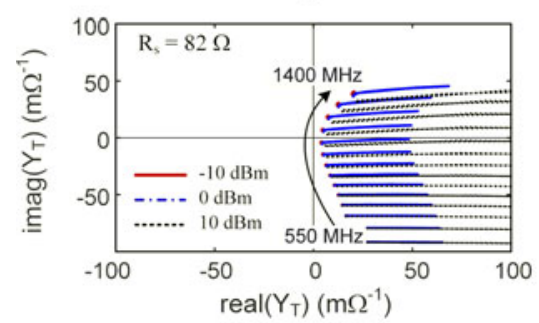

(c)

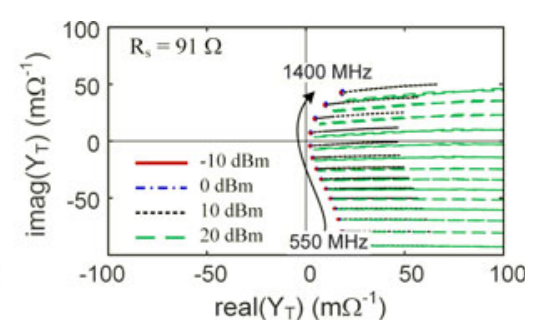

(b)

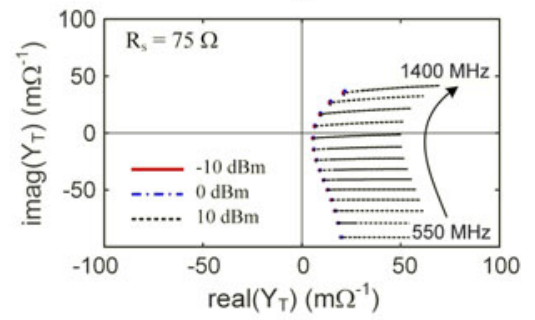

(d)

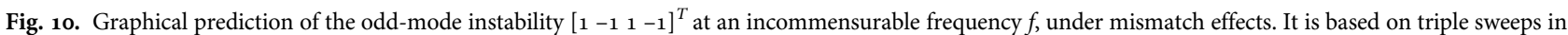
the perturbation frequency $f$ and the reflection-coefficient magnitude $\rho_{o}$ and phase $\phi_{o}$, performed for each $P_{i n}$ value. (a) Stabilization resistor $R_{s}=100 \Omega$. A stability boundary exists within the Smith chart. (b) $R_{s}=91 \Omega$. Tangency situation. (c) $R_{s}=82 \Omega$. There is no instability boundary within the Smith chart. (d) $R_{s}=75 \Omega$. There is no instability boundary within the Smith chart. 


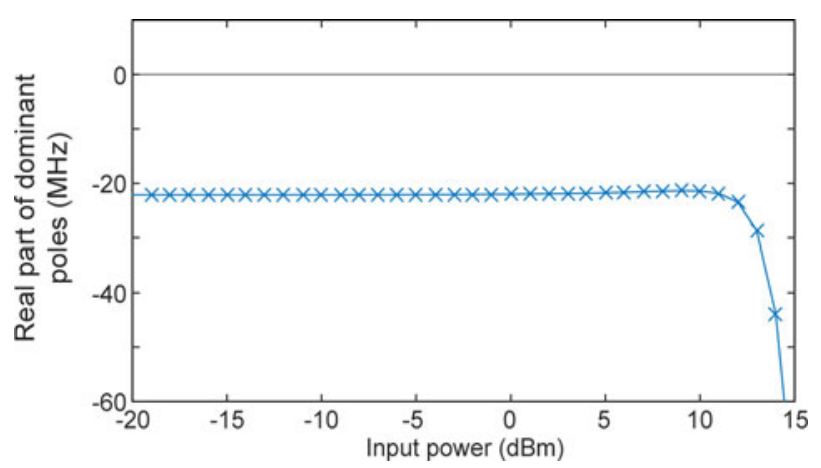

Fig. 11. Pole-zero identification of the matched amplifier (terminated in $50 \Omega$ ), with a stabilization resistor $R_{s}=82 \Omega$. This analysis complements the one based on the admittance diagrams in Fig. 10. Combining the results of the two analyses, it is predicted that the amplifier will be unconditionally stable under mismatch effects for $R_{s}<82 \Omega$.

same situation is obtained for other power values comprised between $P_{\text {in }}=0 \mathrm{dBm}$ and $P_{\text {in }}=10 \mathrm{dBm}$.

Using the described graphical method, in combination with a stability analysis of the matched PA, based on pole-zero identification [8-10], it will be straight forward to stabilize the PA under mismatch effects for all the $P_{\text {in }}$ values. Figure 10(b) shows the same admittance plots in the presence of a stabilization resistor of $R_{s}=91 \Omega$, which nearly corresponds to a tangency condition. Figures $10(\mathrm{c})$ and $10(\mathrm{~d})$ show the admittance plots corresponding to $R_{s}=82 \Omega$ and $R_{s}=75 \Omega$, without any possible fulfillment of the limit oscillation condition $Y_{T o}=0$. Provided that the matched PA (under a $50 \Omega$ termination) is stable for all the $P_{\text {in }}$ values with $R_{s}<82 \Omega$, it will also be unconditionally stable under mismatch effects. The results of the pole-zero identification for $R_{s}=75 \Omega$ are shown in Fig. 11. For all the $P_{i n}$ values, the poles are located on the LHS, so the amplifier must be unconditionally stable under mismatch effects. This result will be experimentally confirmed in Section IV.C.

Departing from an initial point obtained with the plots in Fig. 10, and by means of a continuation method, it is possible to trace the stability boundaries in the $\Gamma_{o}$ Smith chart. For the stabilization resistor $R_{s}=100 \Omega$, this provides the loci shown in Fig. 12, which delimit the termination loads $\Gamma_{o}$ that would give rise to unstable behavior. The impact of $P_{i n}$ on the potential-instability boundary can be noticed. There is a significant variation of the shape and size of the boundaries when modifying $P_{\text {in }}$.

Stable and unstable regions can be distinguished with the aid of the pole-zero identification in Fig. 9, applied to the matched amplifier. For instance, for $P_{\text {in }}=1 \mathrm{dBm}$, the stable region corresponds to the outside of the boundary, since we know that the matched $50 \Omega$ termination is stable and should belong to the stable region. The boundary crosses the origin of the Smith chart at $P_{i n}=2.55 \mathrm{dBm}$ and $P_{\text {in }}=$ 10.9 $\mathrm{dBm}$, which agree with the input power values at which the matched PA undergoes Hopf bifurcations in the pole-zero analysis of Fig. 9.

The accuracy of the stability boundaries in Fig. 12 has been validated with pole-zero identification, considering the two fundamental-frequency loads, $A$ and $B$, one at each side of the boundary corresponding to $P_{i n}=10 \mathrm{dBm}$. For $A$, the poles are on the LHS (Fig. 13), in agreement with the stable behavior predicted by the boundary. For $B$, the poles are on the LHS, also in agreement with the boundary.

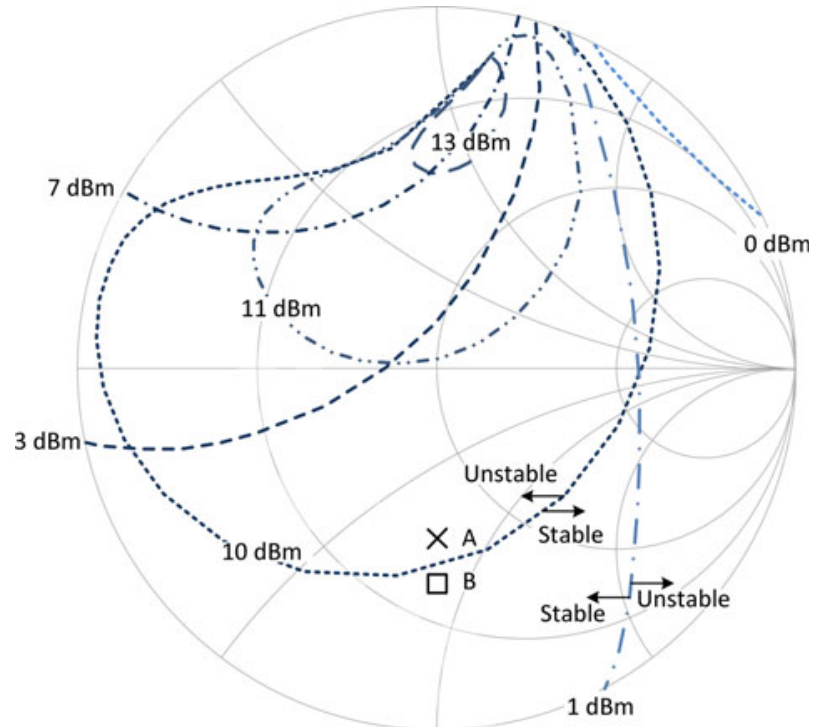

(a)

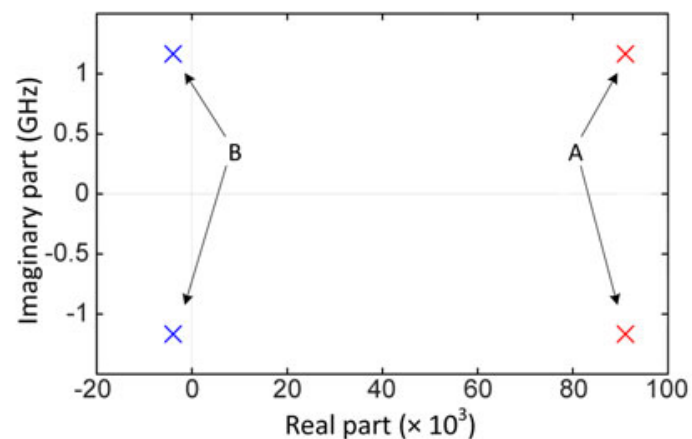

(b)

Fig. 12. Evolution of the instability boundary under incommnesurable oscillations in the odd mode $\left[\begin{array}{llll}1 & -1 & 1 & -1\end{array}\right]^{T}$ under variations in the input power $P_{i n}$. (a) Stability boundaries in the $\Gamma_{o}$ Smith chart. Stable and unstable regions are distinguished with a complementary stability analysis of the matched amplifier (terminated in $50 \Omega$ ) versus $P_{i n}$. This analysis is shown in Fig. 9. (b) Validation of the stability predictions obtained with the boundary corresponding to $P_{\text {in }}=10 \mathrm{dBm}$, through pole-zero identifiction. Two different loads have been tested, $A$ and $B$, indicated in (a). The boundary accurately predicts the stability properties.

\section{2) MODE $\left[\begin{array}{lll}1 & 1 & -1-1\end{array}\right]^{T}$}

To predict potential instabilities in the mode $\left[\begin{array}{llll}1 & 1 & -1 & -1\end{array}\right]^{T}$, four AGs, with the respective phase values $\left(0,0,180,180^{\circ}\right)$, and operating at the incommensurable frequency $f$, are connected at the device output terminals. Though not shown here, all the resulting admittance plots fulfill $\operatorname{Re}\left(Y_{T}\right)>0$ for all the $P_{\text {in }}$ values. Thus, there cannot be any instability boundaries inside the Smith chart. Under this odd-mode excitation, the matched PA is stable for all the $P_{\text {in }}$ values, as verified with pole-zero identification. Taking both results into account, one concludes that the PA does not exhibit this kind of instability.

\section{B) Subharmonic oscillation}

The potential subharmonic oscillation $\left(\right.$ at $\left.f_{i n} / 2\right)$ in each of the two

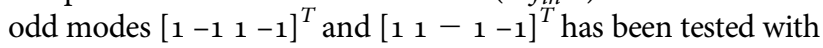
the graphical method. Four AGs with the respective phase distributions: $\left(0,180,0,180^{\circ}\right)$ and $\left(0,0,180,180^{\circ}\right)$ have been connected in parallel at the device output terminals [Fig. 8(a)]. The AG frequencies are $f_{A G}=f_{i n} / 2$. For each of the two AG phase distributions, a triple sweep is performed: in the input- 


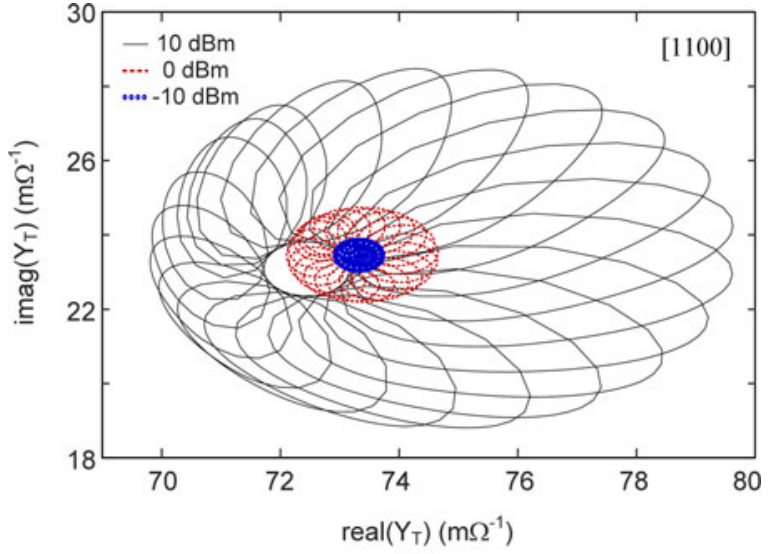

(a)

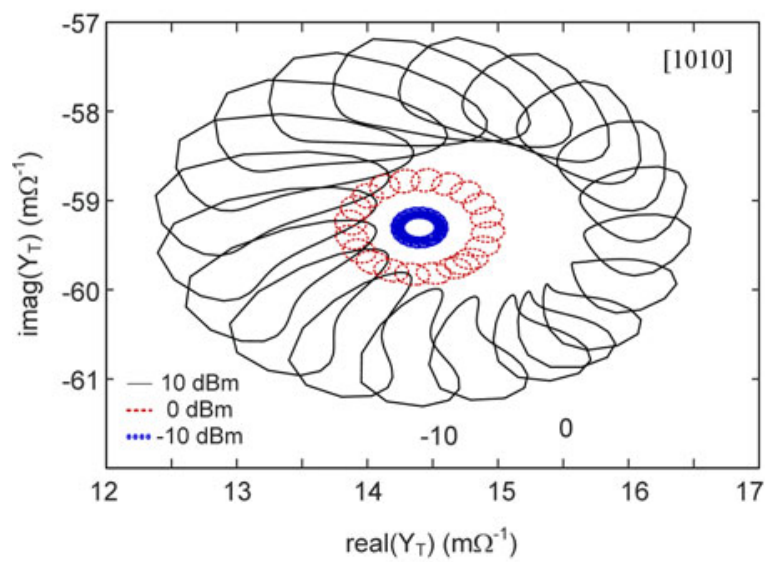

(b)

Fig. 13. Prediction of subharmonic instabilities (at $f_{i n} / 2$ ) under mismatch effects by using a total admittance diagram. Different values of input power $P_{\text {in }}$ have been considered. (a) Odd-mode $\left[\begin{array}{llll}1 & -1 & 1 & -1\end{array}\right]^{T}$, with AG excitation at

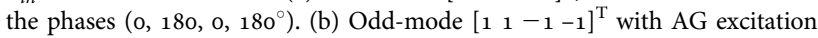
at the phases $\left(0,0,180,180^{\circ}\right)$. For each of the two AG phase distributions, a triple sweep is performed: in the input-source phase $\phi_{i n}$, from o to $360^{\circ}$, and in the amplitude and phase of $\Gamma_{o}$.

source phase $\phi_{i n}$, from o to $360^{\circ}$, and in the amplitude phase of $\Gamma_{o}$. This provides a closed curve for each pair of values $\phi_{i n}, \rho_{o}$. Results obtained for different $P_{\text {in }}$ values are shown in Fig. 13, where the geometrical effect of the double-phase periodicity can be noted. With none of the two excitations, there is a crossing through the negative real semi-axis of the admittance diagram, so there should be no stability boundary in the Smith chart for any $P_{\text {in }}$ value. This information should be complemented with the one obtained through application of pole-zero identification to the matched amplifier. There are no poles on the RHS at the subharmonic frequency for any $P_{i n}$, so the center of the Smith chart is stable. Since there is no instability boundary in the Smith chart for any $P_{i n}$, one concludes that the amplifier is unconditionally stable versus subharmonic instabilities.

\section{C) Experimental results}

The amplifier has been manufactured and measured. Its output was connected to a triple-stub tuner. Under variations of the triple-stub tuner, subharmonic instabilities were only observed through synchronization mechanisms, once the circuit was in a steady-state oscillatory regime. This is a secondary phenomenon, which takes place when the autonomous frequency of the quasiperiodic regime becomes commensurable with the input-drive frequency [18]. From a periodic regime at $f_{\text {in }}$, only transitions to a self-oscillating mixer regime at $f_{\text {in }}$ and $f$ (incommensurable with $f_{\text {in }}$ ) were experimentally observed under variations of the tuner, in agreement with the simulation results. Figure 14 shows the results obtained for the stabilization resistor $R_{s}=$ $100 \Omega$ and $P_{\text {in }}=10 \mathrm{dBm}$. The theoretical stability boundary is represented in Fig. 14(a), together with the measured load-impedance variations obtained for several positions of the triple-stub tuner. Unstable behavior was obtained inside the instability boundary, as in the case of the loads $A$ and $D$. As an example, with the spectrum obtained with the load $A$ is shown in Fig. 14(b). Note that the spectrum in Fig. 8(b) corresponds to the fully established autonomous quasi-periodic regime, exhibiting the oscillation frequency $f_{a}$. In this regime, only the spectral lines $m f_{\text {in }}+k f_{a}$, with $k$ odd are $180^{\circ}$ out of phase. Spectral lines with $k$ even are in phase and are combined by the output network. All the lines comprised between $\mathrm{dc}$ and $f_{\text {in }}$ are more than $30 \mathrm{~dB}$ below the one at $f_{i n}$. The spectral line at $1.85 \mathrm{GHz}$, exhibiting high power, agrees with the second harmonic of one of the autonomously generated line at $0.925 \mathrm{GHz}$. Stable behavior was obtained outside the boundary, as in the case of the loads

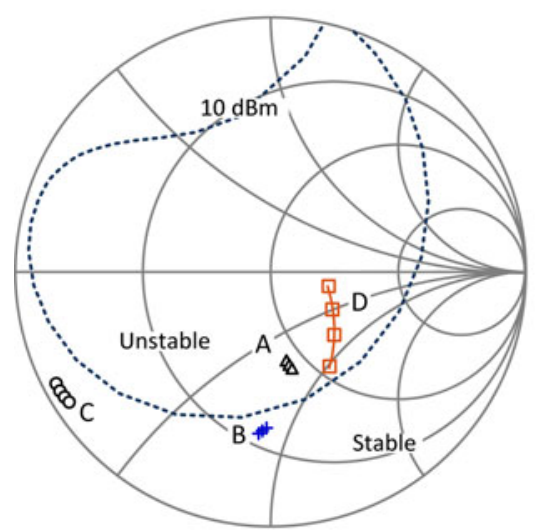

(a)

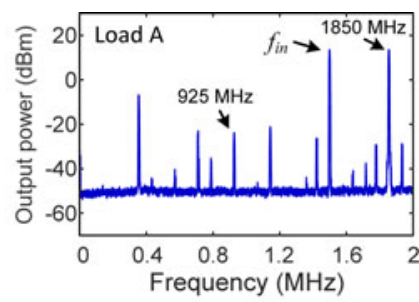

(b)

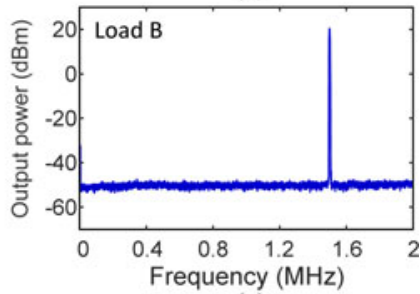

(c)

Fig. 14. Experimental measurement of the PA with four active devices in Fig. 8. (a) Validation of the instability boundary under incommnesurable oscillations in the odd mode $\left[\begin{array}{lll}1 & -1 & 1\end{array}-1\right]^{T}$, corresponding to the input power $P_{\text {in }}=10 \mathrm{dBm}$. (b) Unstable behavior for the experimental load A. (c) Stable behavior for the experimental load B. 
$B$ and $C$. As an example, the spectrum obtained with the load $B$ is shown in Fig. 14(c). When using the resistor value $R_{s}=75 \Omega$, no unstable behavior was obtained for any $P_{\text {in }}$ up to the maximum value $(20 \mathrm{dBm})$, considered in our theoretical analysis.

\section{CONCLUSION}

A method has been presented to predict odd-mode instabilities in PAs under output mismatch effects. The various possible modes are related with the symmetry properties of the matrix describing the active network, calculated with a two-tier conversion-matrix approach. In the case of odd-mode instability, the antenna impedance influences the stability properties only through its value at the fundamental and harmonic frequencies. Under sufficient low-pass filtering effects, the antenna mismatch analysis can be limited to the fundamental frequency. The prediction of odd-mode instabilities consists of two different stages, depending on the desired level of insight into the potential-instability mechanism. The first stage is a graphical method based on admittance diagrams, which must be combined with an ordinary stability analysis of the matched PA (terminated in $50 \Omega$ ) versus the input power. This enables an efficient detection of potential instabilities. The second stage is a calculation of the stability boundaries in the Smith chart corresponding to the termination at the fundamental frequency. The two analyses must be carried out for all the possible odd-mode oscillations and should distinguish the cases of incommensurable and subharmonic oscillations. The method has been applied to two different PA, containing two and four active devices, respectively, which have been manufactured and measured. Very good agreement has been obtained with the simulation results.

\section{ACKNDWLEDGEMENT}

This work has been funded by the Spanish Government under Contract No. TEC2014-60283-C3-1-R, the European Regional Development Fund (ERDF/FEDER) and the Parliament of Cantabria (12.JPo2.64069).

\section{REFERENCES}

[1] Dellier, S.; Gourseyrol, R.; Soubercaze-Pun, G.; Collantes, J.; Anakabe, A.; Narendra, K.: Stability analysis of microwave circuits, in (WAMICON), 2012 IEEE Wireless \& Microwave Technology Conference, Cocoa Beach, FL, 2012, pp. 1-5.

[2] Imbornone, J.F.; Murphy, M.; Donahue, R.S.; Heaney, E.: New insight into subharmonic oscillation mode of GaAs power amplifiers under severe output mismatch condition. IEEE J. Solid State Circuits, 32 (1997), 1319-1325.

[3] Anakabe, A.; Ayllón, N.; Collantes, J.; Mallet, A.; Soubercaze-Pun, G.; Narendra, K.: Automatic pole-zero identification for multivariable large-signal stability analysis of RF and microwave circuits, in Eur. Microwave Conf. (EuMC), Paris, 2010, 477-480.

[4] Suárez, A.; Ramírez, F.; Sancho, S.: Generalized stability criteria for power amplifiers under mismatch effects. IEEE Trans. Microw. Theory Tech., 63 (12) (2015), 4415-4428.

[5] Suárez, A.; Ramírez, F.; Sancho, S.: Stability analysis of power amplifiers under output mismatch effects. IEEE Trans. Microw. Theory Tech., 62 (10) (2014), 2273-2289.
[6] Rollett, J.M.: Stability and power-gain invariants of linear two ports. Inst. Radio Eng. Trans. Circuit Theory, CT-9 (1962), 29-32.

[7] Edwards, M.L.; Sinsky, J.H.: A new criterion for linear 2-port stability using geometrically derived parameters. IEEE Trans. Microw. Theory Tech., 40 (12) (1992), 2303-2311.

[8] Ayllon, N.; Collantes, J.M.; Anakabe, A.; Lizarraga, I.; Soubercaze-Pun, G.; Forestier, S.: Systematic approach to the stabilization of multitransistor circuits. IEEE Trans. Microw. Theory Tech., 59 (8) (2011), 2073-2082.

[9] Jugo, J.; Portilla, J.; Anakabe, A.; Suárez, A.; Collantes, J.M.: Closed-loop stability analysis of microwave amplifiers. IEE Electron. Lett., 37 (4) (2001), 226-228.

[10] Anakabe, A.; Collantes, J.M.; Portilla, J. et al.: Analysis and elimination of parametric oscillations in monolithic power amplifiers, in 2002 IEEE MTT-S Int. Microwave Symp. Digest, Seattle, WA, June 2002, 2181-2184.

[11] Freitag, R.G.: A unified analysis of MMIC power amplifier stability, in 1992 IEEE MTT-S Int. Microwave Symp. Digest, Albuquerque, NM, USA, vol. 1, 1992, 297-300.

[12] Mons, S.; Nallatamby, J.-C.; Queré, R.; Savary, P.; Obregon, J.: A unified approach for the linear and nonlinear stability analysis of microwave circuits using commercially available tools. IEEE Trans. Microw. Theory Tech., 47 (12) (1999), 2403-2409.

[13] Paillot, J.M.; Nallatamby, J.C.; Hessane, M.; Quéré, R.; Prigent, M.; Rousset, J.: A general program for steady state, stability, and FM noise analysis of microwave oscillators, in IEEE MTT Symp., 1990, 1287-1290.

[14] Rizzoli, V.; Mastri, F.; Masotti, D.: General noise analysis of nonlinear microwave circuits by the piecewise harmonic balance technique. IEEE Trans. Microw. Theory Tech., 42 (5) (1994), 807-819.

[15] Quéré, R.; Ngoya, E.; Camiade, M.; Suarez, A.; Hessane, M.; Obregon, J.: Large signal design of broadband monolithic microwave frequency dividers and phase-locked oscillators. IEEE Trans. Microw. Theory Tech., 41 (11) (1993), 1928-1938.

[16] Suárez, A.; Quéré, R.: Stability Analysis of Nonlinear Microwave Circuits, Artech House, Norwood, MA, 2003.

[17] Guckenheimer, J.; Holmes, P.: Nonlinear Oscillations, Dynamical Systems and Bifurcations of Vector Fields, Springer-Verlag, New York, 1990.

[18] Suárez, A.: Analysis and Design of Autonomous Microwave Circuits, IEEE-Wiley, New York, 2009.

[19] Wiggins, S.: Introduction to Applied Nonlinear Dynamical Systems and Chaos, Springer-Verlag, New York, 1990.

[20] Rizzoli, V.; Neri, A.: State of the art and present trends in nonlinear microwave CAD techniques. IEEE Trans. Microw. Theory Tech., 36 (1988), 343-356.

[21] Di Paolo, F.; Leuzzi, G.: Bifurcation synthesis by means of harmonic balance and conversion matrix, in Proc. of the Eur. Gallium Arsenide Applications Symp., Munich, October 2003, 521-524.

[22] Pantoli, L.; Suárez, A.; Leuzzi, G.; Di Paolo, F.: Complete and systematic simulation tools for frequency divider design. IEEE Trans. Microw. Theory Tech., 56 (11) (2008), 2442-2452.

[23] Sarkissian, J.C.; Camiade, M.; Savary, P.; Suárez, A.; Quéré, R.; Obregón, J.: A 6o-Ghz HEMT MMIC analog frequency divider by two. IEEE J. Solid State Circuits, 30 (10) (1995), 1062-1067.

[24] Suárez, A.; Jeon, S.; Rutledge, D.: Stability analysis and stabilization of power amplifiers. IEEE Microw. Mag., 7 (2006), 51-65.

[25] Sancho, S.; Ramírez, F.; Suárez, A.: General stabilization techniques for microwave oscillators. IEEE Microw. Compon. Lett., 15 (2005), 868-870. 